\title{
Inhaltsverzeichnis / Table des matières
}

3 (d) Francis Loser, Peter Voll

6 (f) Editorial / Éditorial

\section{Beiträge / Contributions}

$9 \quad$ Myrian Carbajal et Annamaria Colombo

Postures professionnelles concernant les transactions sexuelles impliquant des jeunes: entre souci de (sur)protection et accompagnement de la socialisation sexuelle

Laura Mellini et Francesca Poglia Mileti

L'agentivité sexuelle au prisme du genre et de la migration: le cas des jeunes issu.e.s de la migration subsaharienne en Suisse

51 Bettina Brüschweiler und Matthias Weber

Organisational eingelassene Professionalitätsansprüche: ein Plädoyer für theoretisch fundierte Einrichtungs- bzw. Angebotskonzepte

\section{Buchbesprechungen / Récensions critiques}

$71 \quad$ Barbara Waldis

Piñeiro, Esteban; Koch, Martina \& Pasche, Nathalie (2021). Un/doing Ethnicity im öffentlichen Dienst. Ethnografien zum ethnischen Differenzieren am Beispiel von Jugendamt und Polizei

$77 \quad$ Stefanie Kurt

Leyvraz, Anne-Cécile; Rey, Raphaël; Rosset, Damian et Stünzi, Robin (2020). Asile et Abus - Regards pluridisciplinaires sur un discours dominant. Asyl und Missbrauch - Multidisziplinäre Perspektiven auf einen vorherrschenden Diskurs 
83 Martina Hörmann

Abplanalp, Esther; Cruceli, Salvatore; Disler, Stephanie; Pulver, Caroline und Zwilling Michael (2020). Beraten in der Sozialen Arbeit. Eine Verortung zentraler Beratungsanforderungen

Neuerscheinungen / Nouvelles Parutions 